\title{
A Very Large, Spontaneous Stratospheric Sudden Warming in a Simple AGCM: A Prototype for the Southern Hemisphere Warming of 2002?
}

\author{
PAUL J. KuSHNER \\ Department of Physics, University of Toronto, Toronto, Ontario, Canada \\ LoRenzo M. Polvani \\ Department of Applied Physics and Applied Mathematics, and the Department of Earth and Environmental Sciences, \\ Columbia University, New York, New York
}

(Manuscript received 19 May 2003, in final form 13 February 2004)

\begin{abstract}
An exceptionally strong stratospheric sudden warming (SSW) that spontaneously occurs in a very simple stratosphere-troposphere AGCM is discussed. The model is a dry, hydrostatic, primitive equation model without planetary stationary waves. Transient baroclinic wave-wave interaction in the troposphere thus provides the only source of upward-propagating wave activity into the stratosphere. The model's SSW is grossly similar to the Southern Hemisphere major SSW of 2002: it occurs after weaker warmings "precondition" the polar vortex for breaking, it involves a split of the polar vortex, and it has a downwardpropagating signature. These similarities suggest that the Southern Hemisphere SSW of 2002 might itself have been caused by transient baroclinic wave-wave interaction. The simple model used for this study also provides some insight into how often such extreme events might occur. The frequency distribution of SSWs in the model has exponential, as opposed to Gaussian, tails. This suggests that very large amplitude SSWs, though rare, might occur with higher frequency than might be naively expected.
\end{abstract}

\section{Introduction}

The austral winter of 2002 was exceptional in the Southern Hemisphere stratosphere because of the major stratospheric sudden warming (SSW) that occurred in September-the only such warming ever observed there-and because of the very warm stratospheric temperatures that occurred in the months leading up to the SSW (Baldwin et al. 2003, hereafter BHOY03; Newman and Nash 2005, hereafter NN05). It is unclear what might have brought about such an exceptional season. BHOY03 argue that extreme events of this kind can occur randomly over a sufficiently long period. NN05 propose a more specific mechanism that involves an initial tropical wave event. One avenue that may lead to an understanding of the Southern Hemisphere SSW of 2002 is to work within the simplest possible modeling framework that still captures the essential dy-

\footnotetext{
Corresponding author address: Dr. Paul J. Kushner, Department of Physics, University of Toronto, 60 St. George St., Toronto ON M5S 1A7, Canada.

E-mail:paul.kushner@utoronto.ca
}

namical features of the coupled extratropical stratosphere-troposphere system. With this idea in mind, we demonstrate here that a very simple model of this system (Polvani and Kushner 2002, hereafter PK; Kushner and Polvani 2004, hereafter KP) is capable of producing an SSW that is similar in many ways to the Southern Hemisphere SSW of 2002, notably, in its surprisingly large amplitude.

\section{Model}

As we have described in detail in previous studies (PK; KP), our model solves the dry, hydrostatic, primitive equations on the sphere, with spectral T42 resolution in the horizontal, 40 levels in the vertical, and a flat lower boundary. The model's winds are linearly damped in a planetary boundary layer and in a sponge above $0.5 \mathrm{hPa}$. The model's temperature field is relaxed toward a zonally symmetric and time-independent equilibrium temperature field, $T_{\text {eq }}$. In the troposphere, $T_{\text {eq }}$ closely follows the Held and Suarez (1994) prescription; in the stratosphere, $T_{\text {eq }}$ transitions from winter polar-night conditions in the winter (southern) hemi- 
sphere to standard atmosphere conditions in the summer (northern) hemisphere. The model's circulation has a jet driven by baroclinic eddies in the troposphere, a tropical overturning circulation, and a stratospheric polar vortex in the winter hemisphere. The strength of the model's polar vortex is governed by a parameter, $\gamma$, that controls the strength of the pole-to-equator gradient in the stratospheric $T_{\text {eq. }}$. We focus here upon the " $\gamma$ $=2$ " case of $\mathrm{PK}$ and $\mathrm{KP}$, which is a case with a relatively weak vortex. The model is integrated for 12000 days, and the last 11000 days are used for analysis. We also briefly consider the $\gamma=4$ case of PK and KP, which is a case with a relatively strong vortex, at the end of section 3.

Because its lower boundary is flat, and because $T_{\text {eq }}$ is zonally symmetric, our model has no stationary planetary waves. Thus, the model's stratospheric variability is driven by transient baroclinic wave-wave interaction alone, as described by Scinocca and Haynes (1998). Our model is thus relevant to the Southern Hemisphere stratosphere-troposphere system, in which driving of stratospheric variability by transient eddies is strong. Our model is simpler than the dry AGCM simulations of Taguchi and Yoden (2002a,b), which include stationary eddies associated with topography in addition to baroclinic eddies. Another simplification compared to the Taguchi-Yoden simulations is that, because $T_{\text {eq }}$ is time independent, our model includes no seasonal cycle. Therefore, our model represents a very simple starting point for understanding the coupled stratosphere-troposphere dynamics underlying SSWs.

\section{Results}

Figure 1 shows the time series, over the 11000 -day analysis period, of the temperature averaged meridionally over a polar cap extending from $90^{\circ}$ to $60^{\circ} \mathrm{S}$ at the 50 -hPa level in the lower stratosphere. The mean value of this quantity is approximately $213 \mathrm{~K}$, and there are many periods when the mean temperature exceeds $216 \mathrm{~K}$. However, we note that there is one event, marked in the figure by a dashed line as occurring at day 7121 , in which the mean temperature exceeds 220 $\mathrm{K}$. The day-7121 event stands out as a departure of over six standard deviations from the time mean. This is illustrated by the right-hand axis in the figure that rescales the data in units of standard deviations from the time mean; that is, the right-hand axis represents the temperature as a nondimensional temperature anomaly:

$$
s(T)=\frac{(T-\bar{T})}{\operatorname{std}(T)},
$$

where $T$ is the meridionally averaged temperature, $\bar{T}$ is the time mean of the time series, and $\operatorname{std}(T)$ is the temporal standard deviation of the time series.

The day-7121 warm anomaly at $50 \mathrm{hPa}$ is a signature of a "major" SSW (as defined by Andrews et al. 1987, p. 259). Figure 2 shows snapshots of the zonal-mean temperature over 6-day intervals starting at day 7115. From day 7115 to day 7121, high-latitude temperatures increase markedly throughout the stratosphere. For ex-

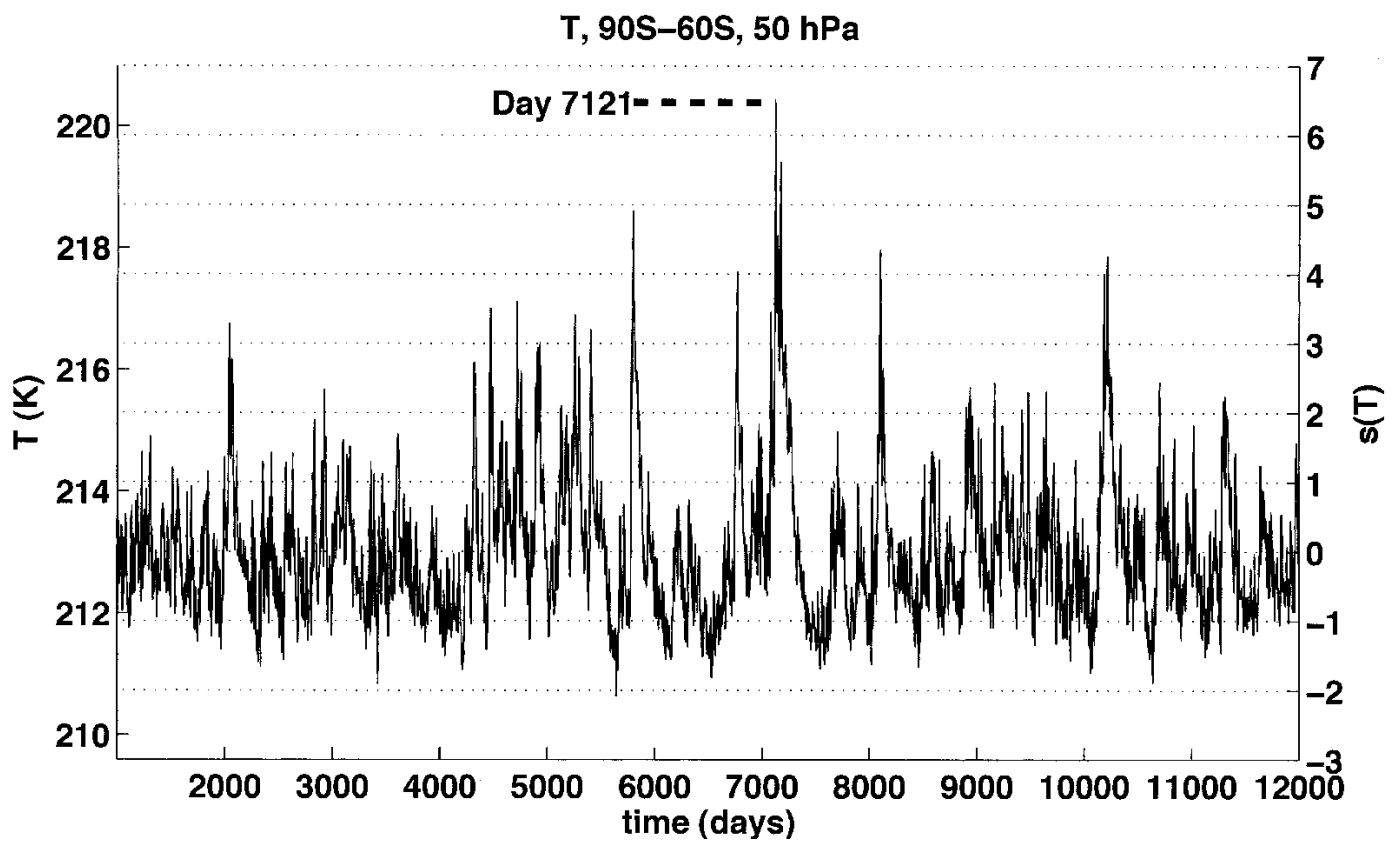

FIG. 1. Time series of 50-hPa temperature averaged from $90^{\circ}$ to $60^{\circ} \mathrm{S}$ in $\mathrm{K}$ (left-hand axis) and in nondimensional anomaly units defined by Eq. (1) (right-hand axis). The day-7121 event is marked with a dashed line. 

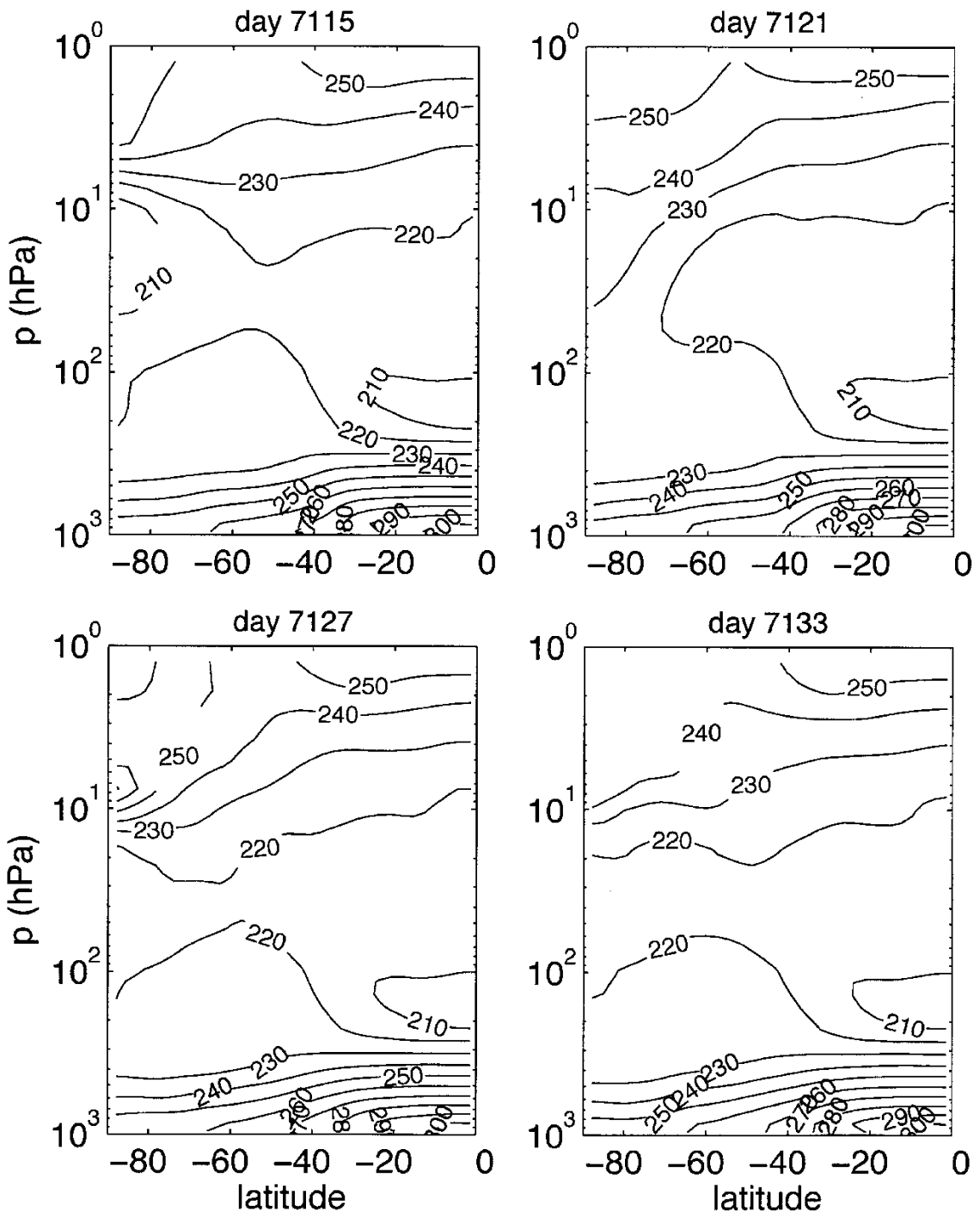

FIG. 2. Southern Hemisphere zonal-mean temperature for 4 days around the time of the day-7121 SSW. The contour interval is $10 \mathrm{~K}$.

ample, the polar temperature at $22 \mathrm{hPa}$ changes from 204 to $235 \mathrm{~K}$ over this 6-day interval. Since low-latitude temperatures do not change as much over the period, the sign of the meridional temperature gradient changes from positive to negative throughout the extratropical stratosphere. Stratospheric temperatures remain warm, and the stratospheric temperature gradient remains negative or weakly positive until day 7133 . The zonal-mean wind evolution (Fig. 3), as expected, follows the temperature evolution closely. Winds switch sign from westerly to easterly from day 7115 to 7121 and then back to weak westerly over the next 12 days.

Figure 4 shows four snapshots of the potential vorticity on the $750-\mathrm{K}$ potential temperature surface (which corresponds approximately to the 10-hPa pres- sure surface), starting at day 7107, instead of day 7115 , to show the less disturbed state of the polar vortex before the warming. At day 7113, the polar vortex is elongated in a manner similar to that seen a few days before typical SSWs, including the Southern Hemisphere SSW of 2002 (BHOY03). The vortex is pinched at its center by day 7119 and is split by day 7125 . This is the only occurrence of a split polar vortex in the entire integration. The complete breakup of the polar vortex is what makes this event qualitatively quite similar to the Southern Hemisphere SSW of 2002, as seen by comparing Fig. 4 to Fig. 2 of BHOY03.

Furthermore, similar to the Southern Hemisphere SSW of 2002 (see Fig. 2 of NN05), the day-7121 SSW in our model is preceded by a series of strong upward Eliassen-Palm (EP) flux pulses from below that warm 

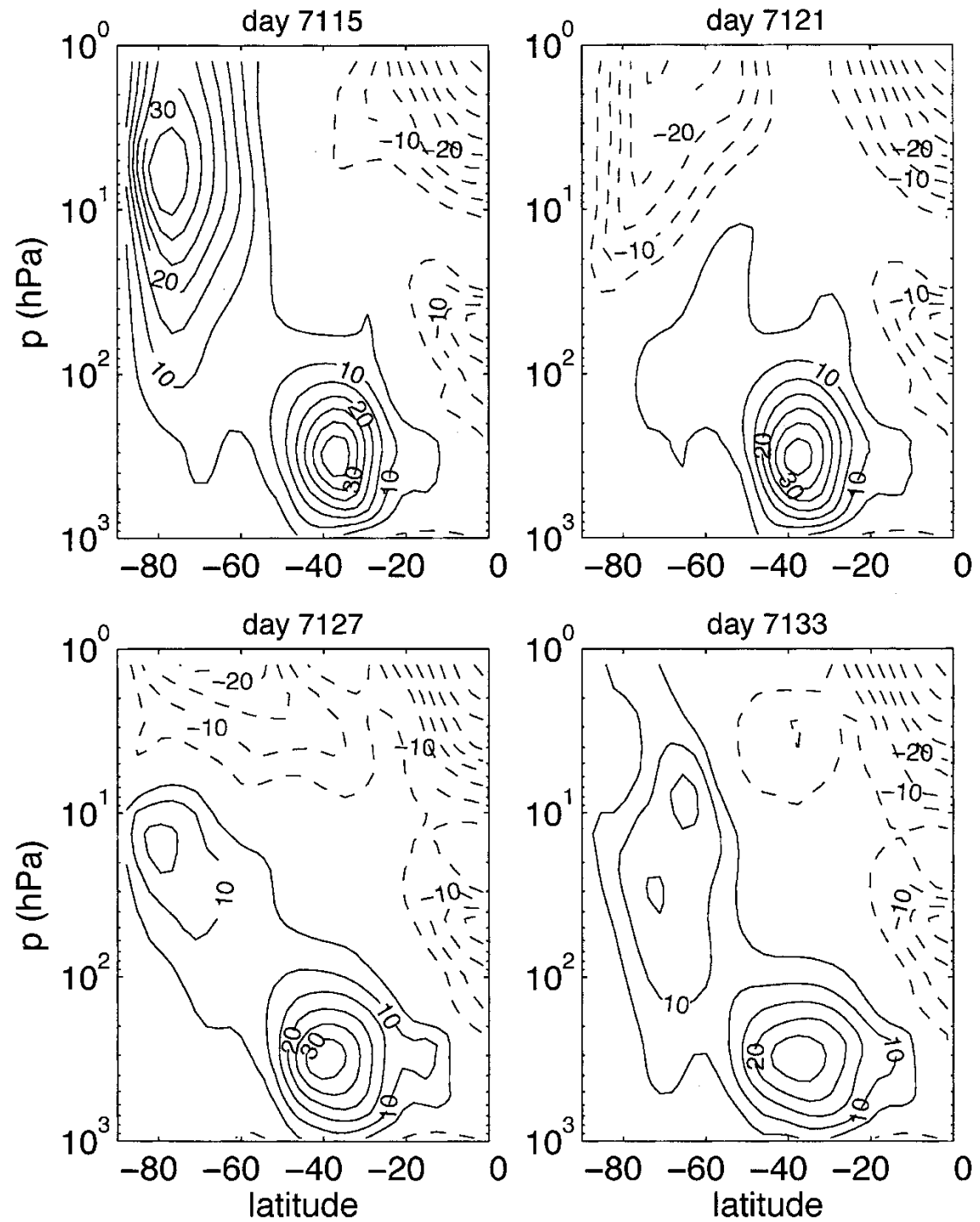

FIG. 3. Same as in Fig. 2, but for the zonal wind. The contour interval is $10 \mathrm{~m} \mathrm{~s}^{-1}$, negative contours are dashed, and the zero contour is omitted.

the stratosphere and weaken the polar vortex. To show this, we plot the $90^{\circ}-60^{\circ} \mathrm{S}$ meridionally averaged temperature anomaly (Fig. 5a), the $90^{\circ}-40^{\circ} \mathrm{S}$ meridionally averaged vertical EP flux anomaly at $100 \mathrm{hPa}$ (Fig. 5b), and the $90^{\circ}-40^{\circ} \mathrm{S}$ meridionally averaged zonal-wind anomaly (Fig. 5c). All quantities are plotted as nondimensional anomalies similar to Eq. (1), that is, as $s(\cdot)=$ $[(\cdot)-\overline{(\cdot)}] / \operatorname{std}(\cdot)$.

The day-7121 SSW, denoted by the arrow in Fig. 5a, occurs after the stratosphere has been mildly warm for 200 days. This warm period is sustained by strong upward pulses of EP wave activity-several of these events are marked with yellow lines in each of the figure panels. The day-7121 SSW has an interesting precursor: at around day 7080, a particularly strong (4 std dev) pulse of wave activity brings about an 8 std dev warming in the upper stratosphere whose impacts on the winds are confined to above $20 \mathrm{hPa}$. In this event, the polar vortex is greatly deformed but does not split (not shown). This appears to precondition the vortex so that the somewhat weaker wave activity pulse just before the day-7121 event can produce the major warming. Polvani and Saravanan (2000) report a similar effect of preconditioning on stratospheric wave breaking in the presence of closely separated pulses of wave activity.

As is common in major warmings in the Northern Hemisphere, the Southern Hemisphere SSW of 2002 exhibited downward propagation (NN05). Similarly, Fig. 5a shows that, after the day-7121 SSW in our model, anomalously warm temperatures descend into the troposphere, at least to $200 \mathrm{hPa}$, and perhaps all the 

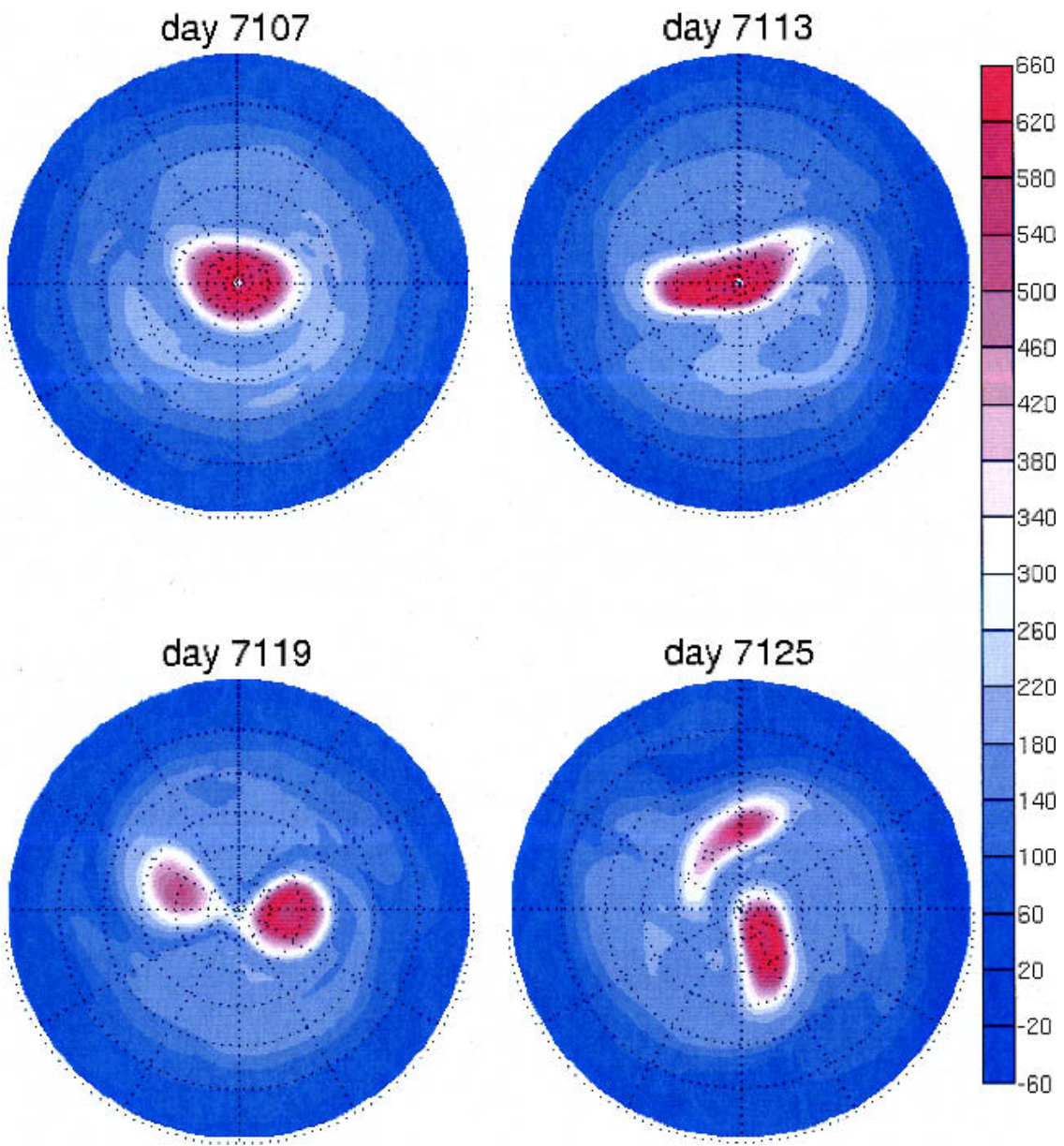

FIG. 4. Snapshots of potential vorticity on the $750-\mathrm{K}$ isentropic surface around the time of the day-7121 SSW. The color shading key is in potential vorticity units.

way to the surface, by day $7250 .{ }^{1}$ The easterly anomaly in the winds, over the wider region $90^{\circ}-40^{\circ} \mathrm{S}$ (Fig. 5c), descends but does not penetrate into the troposphere. Figure 2 of NN05 also shows, similar to this figure, that the temperature signature of the observed SSW descends more deeply into the troposphere than the zonal-wind signature.

Notice, also, similar to Fig. 2a of NN05, that after the day-7121 SSW, a cold-temperature anomaly descends from 1 to $20 \mathrm{hPa}$ as the warm-temperature anomaly descends below it. In Fig. 5c, we see a descending strong-wind anomaly above the descending weak-wind anomaly. These descending patterns are reminiscent of a QBO-like wave-mean flow interaction, along the lines proposed by Plumb and Semeniuk (2003) to ex-

\footnotetext{
${ }^{1}$ The statistical significance of the signal in the lower troposphere is difficult to assess. By day 7200, the lower troposphere has been anomalously cool for several hundred days and may be about to become anomalously warm, independently of what is happening in the stratosphere.
}

plain downward-propagating stratosphere-to-troposphere signals in the Northern Hemisphere (Baldwin and Dunkerton 1999, 2001). The picture is that the breaking level of planetary wave activity absorption is being eroded downward, resulting in a descending region above the breaking level, which is being shielded from upward-propagating wave activity.

The fact that the day-7121 SSW represents a 6 std dev event is, perhaps, not surprising, given the population of warm events that occur during the integration. In this model, as in other models and in the observations, stratospheric temperature variability is skewed and exponentially distributed (Taguchi and Yoden 2002a,b). Figure 6a plots the histogram of the $s(T)$ time series in Fig. 1 with a logarithmic scale on the vertical axis. The warm events are exponentially distributed, as shown by a fit of an exponential function to the data for $s(T) \geq 0$ (see caption for details). The day-7121 SSW is located at the extreme right tail of the distribution and appears as a reasonable end point for the distribution rather than as an unexpected extreme value. 

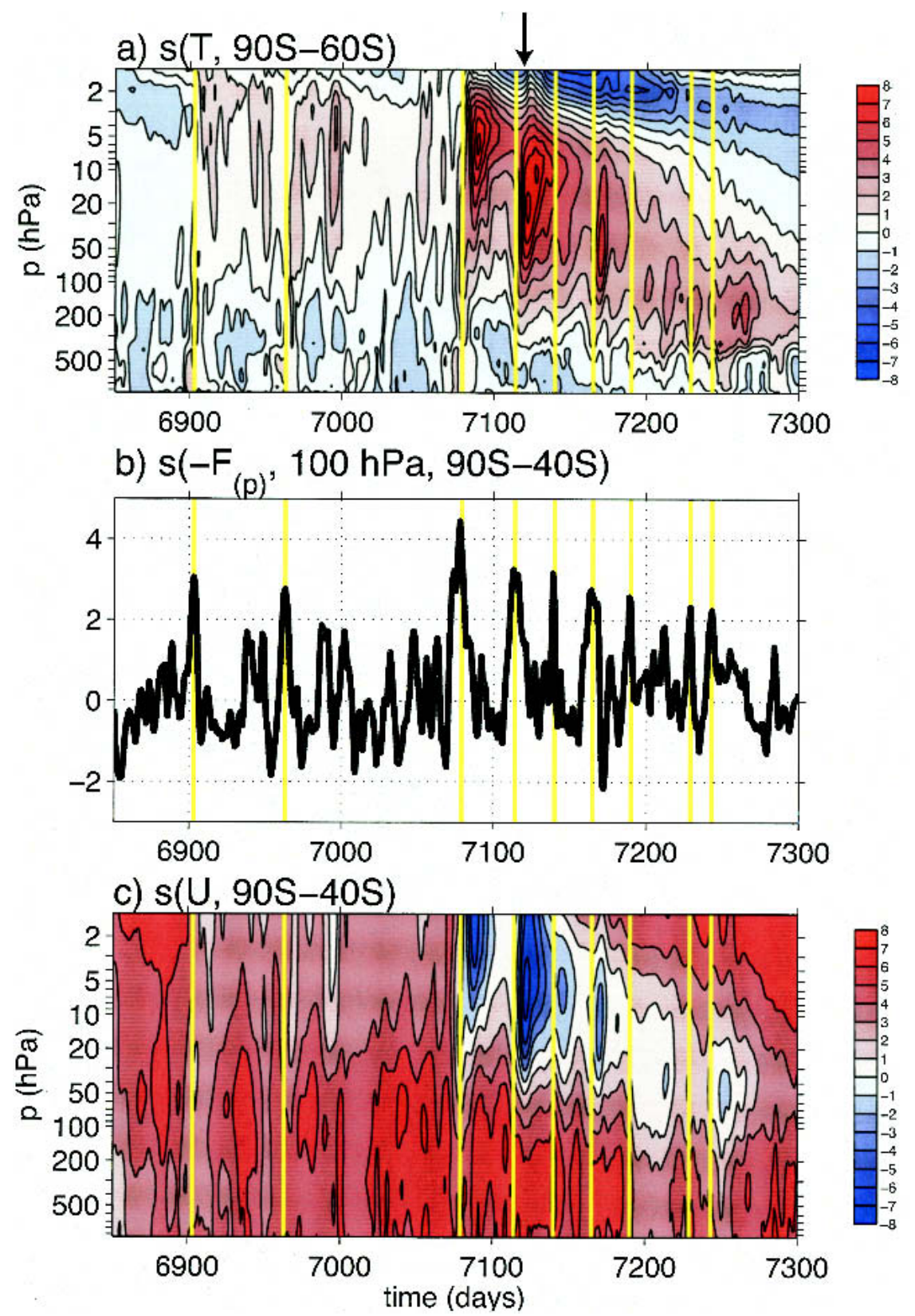

FIG. 5. (a) The $90^{\circ}-60^{\circ} \mathrm{S}$ meridionally averaged temperature anomaly field, defined as in Eq. (1), as a function of pressure and time. Shading interval is $1 \mathrm{std}$ dev. The black arrow denotes the time of the day-7121 SSW. (b) The $90^{\circ}-40^{\circ} \mathrm{S}$ meridionally averaged vertical component of the EP flux at $100 \mathrm{hPa}$, in anomaly units as in (a). (c) Same as in (a), but for $90^{\circ}-40^{\circ} \mathrm{S}$ meridionally averaged zonal-mean zonal wind. Periods when the vertical EP flux in (b) peaks at values greater than +2 std dev are marked with yellow lines.

Finally, one might ask how the likelihood of extreme events such as the day-7121 SSW would change if the stratosphere-troposphere system were externally perturbed, for example, under some climate change scenario. It has been noted (e.g., Khatiwala et al. 2001) that when a distribution of events is exponential, as is the case here, a change in the slope of the distribution is likely to have an important effect, since the "tails" in that case contain many more extreme events than in the case of a Gaussian distribution.

In the context of our simple model, we have attempted to address this question by considering how the distribution of temperature anomalies depends on the parameter $\gamma$. As explained above, this param- 

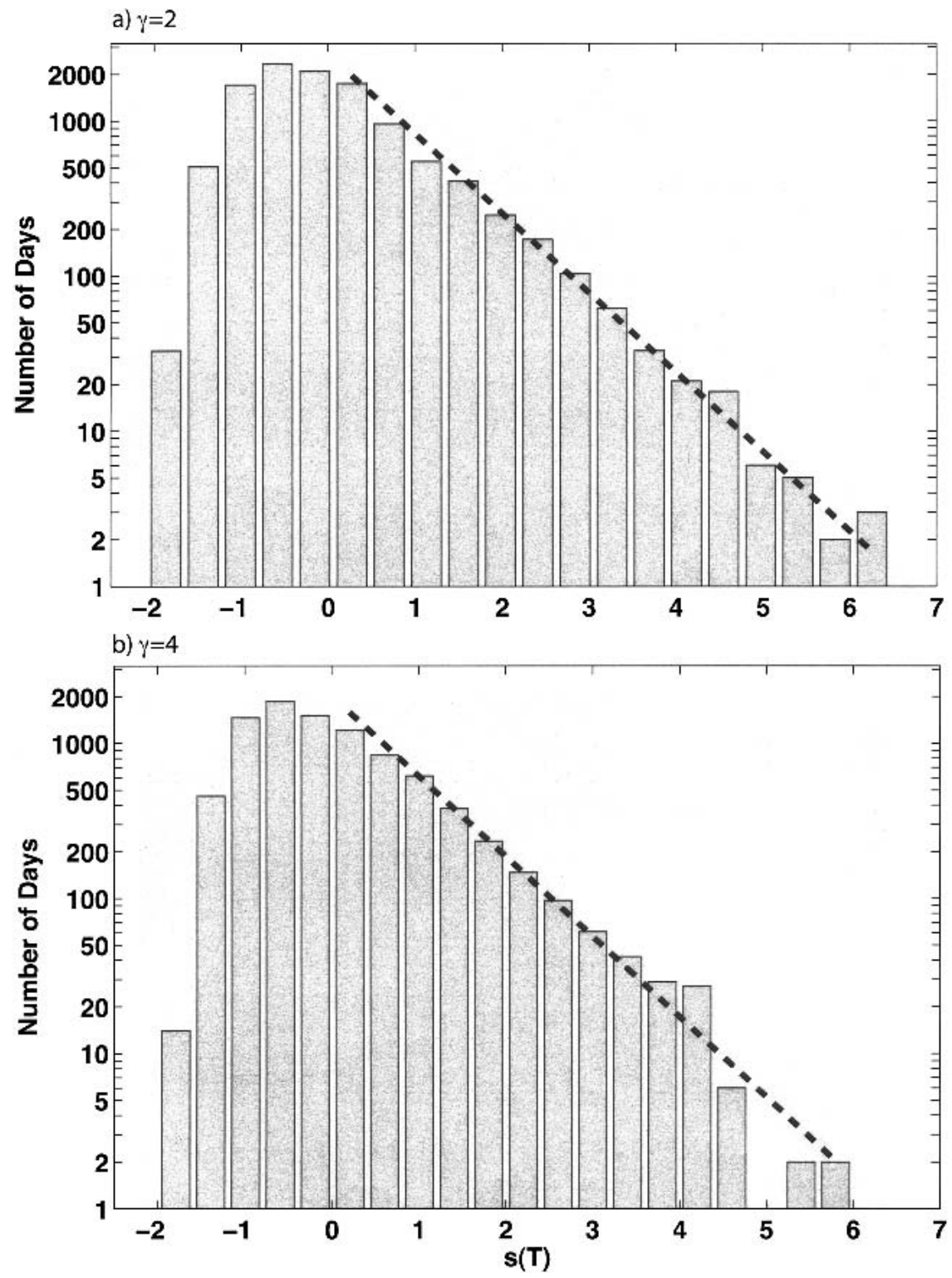

FIG. 6. (a) The bars show the number distribution of $s(T)$ in Fig. 1 for the $\gamma=2$ case. The dashed line is the exponential function $10^{-0.51 s(T)+3.43}$, plotted for $s(T)>0$. The constants in this exponential function were obtained from a best-fit estimate using linear regression; the Pearson $r^{2}$ coefficient for the fit is 0.68 . (b) Same as in (a), but for the $\gamma=4$ case (using a 9000-day analysis period). The dashed line is the exponential function $10^{-0.52 s(T)+3.31}$, plotted for $s(T)>0$, again obtained from linear regression. The Pearson $r^{2}$ coefficient for the fit is 0.58 .

eter largely controls the overall stratospheric temperature and can thus be used to perturb the system (see PK and KP). For the $\gamma=4$ case, corresponding to a stratosphere-troposphere system with a much stronger polar vortex, reduced planetary wave driving, and generally lower stratospheric temperatures, the histogram of temperature anomalies is shown in Fig. 6b. Perhaps surprisingly, we find that the slope (see caption for details) is very nearly identical to the $\gamma=2$ case.
However, it is important to note that for the $\gamma=4$ case, the variance decreases to $\operatorname{std}(T)=0.86 \mathrm{~K}$ from a value of $1.15 \mathrm{~K}$ for $\gamma=2$. Hence, if the temperature anomalies are normalized relative to the "unperturbed" $\gamma=2$ value of $\operatorname{std}(T)$, the slope in Fig. 6b would in fact be steeper, with a corresponding decrease in extreme events thus defined. Whether these findings are peculiar to our simple model or are a property of climate models in general we do not know. Clearly this issue deserves further investigation. 


\section{Conclusions}

We have demonstrated that a very simple model of the Southern Hemisphere stratosphere that is forced only by tropospheric baroclinic eddies can give rise, spontaneously, to an SSW that is similar, in many important ways, to the observed Southern Hemisphere SSW of September 2002. We agree with BHOY03 that, given the skewed distribution of warming temperatures in the observations, in this model and in other models (Taguchi and Yoden 2002a,b), it is likely that extreme events like this one will occur over sufficiently long time periods as a result of random fluctuations in the coupled stratosphere-troposphere system. Therefore, there may be no need to invoke more complicated models to explain the 2002 event.

In closing, we point out that our model's day-7121 SSW was not the result of an attempt on our part to deliberately simulate the 2002 event. Instead, our model's extreme SSW occurred spontaneously, and the main reason to document it is that it resembles so closely, albeit fortuitously, the 2002 event.

Acknowledgments. PJK acknowledges the generous support of NOAA/GFDL, Princeton, New Jersey, for this research. The work of LMP is funded in part by the U.S. National Science Foundation.

\section{REFERENCES}

Andrews, D. G., J. R. Holton, and C. B. Leovy, 1987: Middle Atmosphere Dynamics. International Geophysical Series, Vol. 40, Academic Press, 489 pp.

Baldwin, M. P., and T. J. Dunkerton, 1999: Propagation of the Arctic Oscillation from the stratosphere to the troposphere. J. Geophys. Res., 104, 30 937-30946.
—, and —, 2001: Stratospheric harbingers of anomalous weather regimes. Science, 294, 581-584.

$\longrightarrow$ T. Hirooka, A. O'Neill, and S. Yoden, 2003: Major stratospheric warming in the Southern Hemisphere in 2002: Dynamical aspects of the ozone hole split. SPARC Newsletter, No. 20, SPARC Office, Toronto, ON, Canada, 24-26. [Available online at http://www.atmosp.physics.utoronto.ca/ SPARC/Newsletters.html.]

Held, I. M., and M. J. Suarez, 1994: A proposal for the intercomparison of the dynamical cores of atmospheric general circulation models. Bull. Amer. Meteor. Soc., 75, 1825-1830.

Khatiwala, S., B. Shaw, and M. Cane, 2001: Enhanced sensitivity of persistent events to weak forcing in dynamical and stochastic systems: Implications for climate change. Geophys. Res. Lett., 28, 2633-2636.

Kushner, P. J., and L. M. Polvani, 2004: Stratosphere-troposphere coupling in a relatively simple AGCM: The role of eddies. $J$. Climate, 17, 629-639.

Newman, P. A., and E. R. Nash, 2005: The unusual Southern Hemisphere stratosphere winter of 2002. J. Atmos. Sci., 62, 614-628.

Plumb, R., and K. Semeniuk, 2003: Downward migration of extratropical zonal wind anomalies. J. Geophys. Res., 108, 4223, doi:10.1029/2002JD002773.

Polvani, L. M., and R. Saravanan, 2000: The three-dimensional structure of breaking Rossby waves in the polar wintertime stratosphere. J. Atmos. Sci., 57, 3663-3685.

- , and P. Kushner, 2002: Tropospheric response to stratospheric perturbations in a relatively simple general circulation model. Geophys. Res. Lett., 29, 181-184.

Scinocca, J. F., and P. H. Haynes, 1998: Dynamical forcing of stratospheric planetary waves by tropospheric baroclinic eddies. J. Atmos. Sci., 55, 2361-2392.

Taguchi, M., and S. Yoden, 2002a: Internal interannual variations of the troposphere-stratosphere coupled system in a simple global circulation model. Part I: Parameter sweep experiment. J. Atmos. Sci., 59, 3021-3036.

- , and - 2002b: Internal interannual variations of the troposphere-stratosphere coupled system in a simple global circulation model. Part II: Millennium integrations. J. Atmos. Sci., 59, 3037-3050. 